\title{
Single-Shot Phase-Shifting Digital Holography Based on the Spatial Carrier Interferometry and Its Tolerance Analysis
}

\author{
Yasuhiro Harada, Aizuddin Wan and Hiroyasu Sone \\ Kitami Institute of Technology \\ Japan
}

\section{Introduction}

In digital holography (Kreis, 2005; Schnars \& Jueptner, 2005), holograms are recorded using an image sensor such as CCD cameras, and reconstruction of images of the object is performed by a computer. From this, digital holography has following attractive features: A wet process for developing recording media is not required; qualitative evaluation is easy for three-dimensional images of objects; and focused images of three-dimensional objects at a desired depth can be instantaneously acquired without a mechanical focusing process. However, this technology suffers from lower resolution of image sensors than photographic materials, and therefore, object size to be recorded is restricted to be large and far from the image sensor in off-axis recording geometry. These limitations can be moderated by introducing the phase-shifting method (Schreiber et al., 2007) that enables us to derive complex amplitude of the object wave on the image sensor directly from three or more phase-shifted holograms recorded in the in-line geometry (Yamaguchi \& Zhang, 1997). However, since this method requires to record at least three or four phase-shifted hologram, it is useless for instantaneous measurement of the dynamic object.

In order to resolve this problem, various single-shot technologies with phase-shifting features in subsequent holograms are proposed(Awatsuji et al., 2004; Nomura et al., 2006; Toge et al., 2008; Wyant, 2003). All of these methods are based on space-division multiplexing of the phase-shifted holograms in the single recording but device and technique used are different each other. In the former three methods, special micro-element arrays of phase retarders (Awatsuji et al., 2004) or polarizers (Nomura et al., 2006; Wyant, 2003) suitable to a CCD array for recording are strictly required, and therefore, any satisfactory result for practical instrumentations has not been reported until now. On the other hand, the last method only require an introduction of off-axis plane wave illumination to an image sensor for recording single holograms (Toge et al., 2008). From its simplicity, this method seems to be one of most practical candidate for single-shot digital holography. However, it should be point out that we must align the incident angle of the reference wave plane wave so that the relative phase difference between adjacent pixels of the image sensor becomes $2 \pi / 3$ or $\pi / 2$ radian. This means that very rigorous alignment in reference wave illumination is required. Furthermore, this requirement may be more serious problem in the case of color digital holography where multicolor laser light is utilized. In this situation, optical alignment should be independently 
performed for each wavelength but there are no description about care of the angle of incidence of each reference wave (Toge et al., 2008).

In the present chapter, we concentrate our attention on the problem of the tolerance of the incidence angle of the plane reference wave in the method proposed by (Toge et al., 2008). We theoretically analyze the problem in the Fourier domain using the principle of single-shot phase-shifting digital holography based on the spatial carrier interferometry (Harada et al., 2008 ; 2009). From our theoretical analysis, importance in the alignment of the plane reference wave is brought to light and special procedure to be applied under the situation, where the required condition for the incident angle of the plane reference wave is not satisfied, is given.

\section{Theoretical analysis of the single-shot scheme}

Figure 1 (a) shows a schematic diagram of optical geometry for recoding holograms in the method proposed by (Toge et al., 2008). It is just a off-axis geometry of digital holography where $\theta$ is an incident angle of the plane reference wave, $\lambda$ the wavelength of the light in the holographic recording. We assume the interval of photodiodes in an image sensor is $d$. If the incident angle, $\theta$, of the reference plane wave satisfies the equation given by

$$
\frac{\lambda}{\sin \theta}=3 d \quad \text { or } \quad \sin \theta=\lambda / 3 d
$$

phase of the reference wave along a surface of the image sensor changes periodically with spatial interval of $3 d$. Therefore, the single-shot fringe pattern-hologram-recorded by the image sensor can be considered as a sum of three phase-shifted sub-holograms with constant phase difference of $2 \pi / 3$ in the reference waves as shown in Fig. 1 (b). Therefore, after interpolating the missing data point in each sub-hologram, complex amplitude of the object wave at the image sensor can be reconstructed by using three-step algorithm of phase-shifting interferometry (Schreiber et al., 2007).

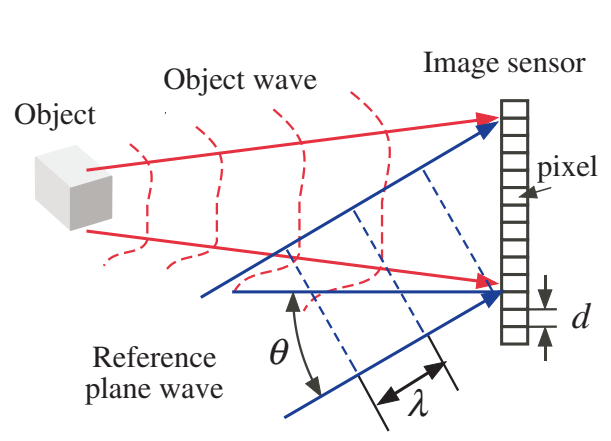

(a) Recording geometry

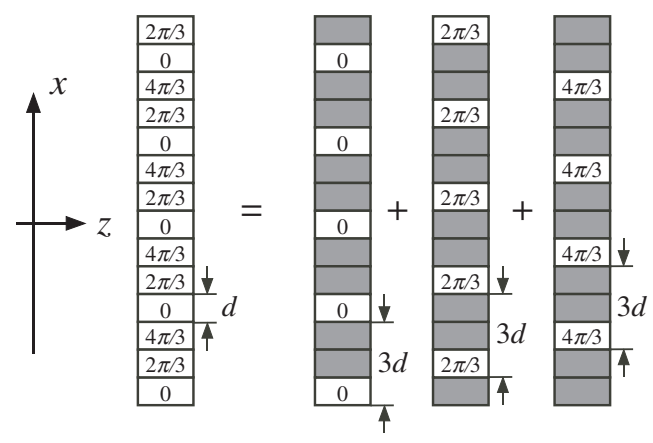

(b) resultant phase distributions at the image sensor under the condition of $\lambda / \sin \theta=3 d$

Fig. 1. Schematic diagrams of recording geometry (a) and resultant phase distributions at the image sensor under the condition of $\lambda / \sin \theta=3 d(b)$. 


\subsection{Hologram intensity distribution and its spectral distributions}

Now let us consider mathematical expression of interference fringe on the image sensor and its sampled version -digital hologram intensity-obtained by sampling detection by the image sensor. If we represent object and reference waves at the image sensor plane by

$$
\begin{aligned}
& U_{O}(x, y)=a(x, y) \exp \{i \phi(x, y)\}, \\
& U_{R}(x, y)=A \exp \left\{i 2 \pi \xi_{0} x\right\},
\end{aligned}
$$

respectively, the hologram intensity, $I(x, y)$, at the image sensor is given by

$$
\begin{aligned}
I(x, y) & =\left|U_{R}(x, y)+U_{O}(x, y)\right|^{2} \\
& =A^{2}+a^{2}(x, y)+2 A a(x, y) \cos \left\{2 \pi \xi_{0} x+\phi(x, y)\right\} \\
& =b(x, y)+c(x, y) \exp \left(i 2 \pi \xi_{0} x\right)+c^{*}(x, y) \exp \left(-i 2 \pi \xi_{0} x\right)
\end{aligned}
$$

where

$$
\begin{aligned}
b(x, y) & =A^{2}+a^{2}(x, y), \\
c(x, y) & =\frac{1}{2} A a(x, y) \exp \{i \phi(x, y)\}, \\
\xi_{0} & =\frac{\sin \theta}{\lambda},
\end{aligned}
$$

respectively. $\xi_{0}$ means a carrier frequency in the interferogram introduced by the oblique incidence of the plane reference wave. One-dimensional Fourier transform of $I(x, y)$ with regard only to $x$ axis gives

$$
\tilde{I}(\xi, y) \equiv \mathcal{F}_{x}[I(x, y)]=B(\xi, y)+C\left(\xi-\xi_{0}, y\right)+C^{*}\left(\xi+\xi_{0}, y\right)
$$

where $\tilde{I}(\xi, y)=\mathcal{F}_{x}[I(x, y)], B(\xi, y)=\mathcal{F}_{x}[B(x, y)]$, and $C(\xi, y)=\mathcal{F}_{x}[c(x, y)]$, respectively. From this expression, it is clear that information of the object wave is mainly stored in the term $C(\xi, y)$ shifted by the carrier frequency $\xi_{0}=\sin \theta / \lambda$ in the spectral domain. Figure 2(a) shows its schematic representation.

\subsection{Image sensor as a sampling array and its spectral distributions}

In digital holography, hologram intensity distribution is detected by an image sensor where the detection of light intensity is performed as spatial sampling by the photodiode in the image sensor. Therefore image sensor can be considered as the sampling array with interval same as the pixel interval. Therefore a mathematical expression for this sampling array can given in ideal case (Gaskill, 1978) by

$$
s(x, y)=\sum_{m} \sum_{n} \delta(x-n d) \delta(y-m d),
$$

and its one-dimensional Fourier transform is given by

$$
\begin{aligned}
\tilde{S}(\xi, y)=\mathcal{F}_{x}[s(x, y)] & =\sum_{m} \sum_{n} \exp (2 \pi n d \xi) \delta(y-m d)=\sum_{m} \sum_{n} \exp \left(\pi \frac{n}{\xi_{N}} \xi\right) \delta(y-m d), \\
& =\sum_{m} \sum_{n} \delta\left(\xi-\frac{n}{d}\right) \delta(y-m d)=\sum_{m} \sum_{n} \delta\left(\xi-n 2 \xi_{N}\right) \delta(y-m d),
\end{aligned}
$$


where $\xi_{N}=1 / 2 d$ is the Nyquist frequency of the image sensor. Figure 2(b) shows its schematic representation.

(a)

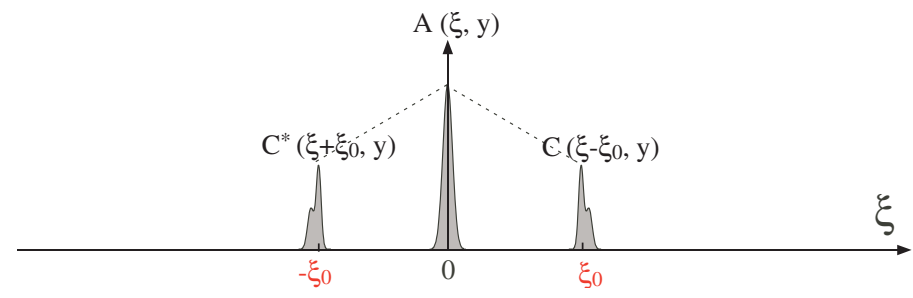

(b)

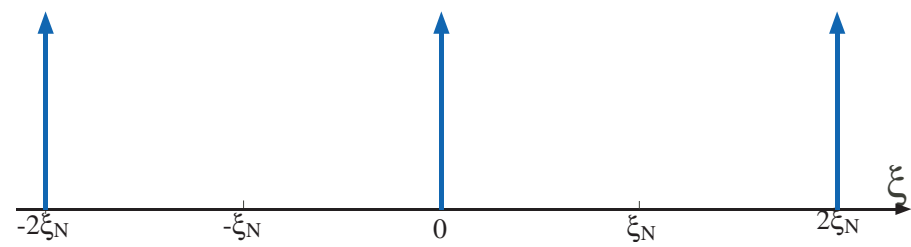

(c) $-2 \xi_{\mathrm{N}}$

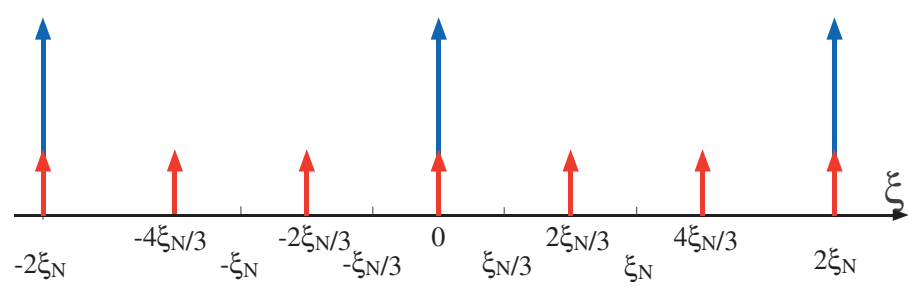

Fig. 2. Spectral distribution of interference fringes and pixel arrays of the image sensor. (a) spectral distribution of the interference fringe at the image sensor, (b) spectral distribution of a pixel array of the image sensor with spatial interval of $d$, and (c) that of a pixel array with the spatial interval of $3 d$, respectively. Here $\xi_{N}=1 / 2 d$ is Nyquist frequency of the original pixel array of the image sensor.

Since the original sampling array of the image sensor with the interval of $d$ in $x$ direction can be recognized as a sum of sparse sampling arrays with an extended interval of $3 d$ and spatial shift of detecting point by $d$, Eqs.(9) and (10) can be rewritten as

$$
s(x, y)=\sum_{j=0}^{2} s^{(j)}(x, y)=\sum_{j=0}^{2} \sum_{n^{\prime}} \sum_{m} \delta\left(x-n^{\prime} 3 d-j d\right) \delta(y-m d),
$$

and

$$
\begin{aligned}
\tilde{S}(\xi, y)=\sum_{j=0}^{2} \tilde{S}^{(j)}(\xi, y) & =\frac{1}{3 d} \sum_{j=0}^{2} \sum_{n^{\prime}} \sum_{m} \exp \left[-i 2 \pi n^{\prime} \frac{j}{3}\right] \delta\left(\xi-\frac{n^{\prime}}{3 d}\right) \delta(y-m d), \\
& =\frac{2 \xi_{N}}{3} \sum_{j=0}^{2} \sum_{n^{\prime}} \sum_{m} \exp \left[-i 2 \pi n^{\prime} \frac{j}{3}\right] \delta\left(\xi-n^{\prime} \frac{2 \xi_{N}}{3}\right) \delta(y-m d),
\end{aligned}
$$


respectively. Schematic representation of absolute value of the spectral distribution $\tilde{S}(j)$ is shown in Fig. 2(c). Please note that, in addition to the widen interval of $3 d$, the shift of the detecting point $j d$ is introduced in the spatial domain representation of Eq. (11). These two differences result in reduced interval of spectral array with $1 / 3 d=2 \xi_{N} / 3$ and new phase term whose phase value depends on the number $j$ and spectral order $n^{\prime}$ in the spectral domain representation of Eqs. (12) and (13). For the sub-detector array $s^{(0)}(x, y)$, the additional phase for spectral array is 0 for all impulsive spectrum of the order $n^{\prime}$ since $j=0$. In contrast to this, for the sub-detector array $s^{(1)}(x, y)$ and $s^{(2)}(x, y)$ result in the impulsive spectral arrays $\tilde{S}^{(1)}(\xi, y)$ and $\tilde{S}^{(2)}(\xi, y)$ with the phase of $-(2 \pi / 3) n^{\prime}$ and $-(4 \pi / 3) n^{\prime}$, respectively. As will be shown in the next subsection this phase term plays very important role in the method for single-shot phase-shifting digital holography (Harada et al., 2008; 2009).

\subsection{Digital holograms detected by the image sensor and its spectral distributions}

Let us consider the digital hologram detected by the image sensor of Eq. (9) and its sub-holograms which correspond to the hologram considered to be detected by individual sparse sampling array, $s^{(j)}(x, y)$, given by Eq. (11). Mathematical expression of the digital hologram, $I_{S}(x, y)$, detected by the image sensor is simply given by a product of the hologram intensity, $I(x, y)$, given by Eq. (4), and the sampling array, $s(x, y)$, given by Eq. (9). Therefore, spectral expression of the digital hologram, $\tilde{I}(\xi, y)$ can be given by the convolution integral between their Fourier spectra as follows:

$$
\tilde{I}_{S}(\xi, y)=\tilde{I}(\xi, y) * \xi \tilde{S}(\xi, y),
$$

where $* \xi$ stands the convolution integral with regard to the variable $\xi$.

In the same manner and with help of linearity shown in Eq. (11), mathematical expressions for the digital sub-holograms, $I_{S}^{(j)}(x, y)(i=0,1,2)$, and their one-dimensional Fourier transform, $\tilde{I}_{S}^{(j)}(\xi, y)$ are given by

$$
\begin{aligned}
& I_{s}^{(j)}(x, y)=I(x, y) \cdot s^{(j)}(x, y), \\
& \tilde{I}_{s}^{(j)}(\xi, y)=\tilde{I}(\xi, y) * \tilde{S}^{(j)}(\xi, y) .
\end{aligned}
$$

By substituting Eqs. (8) and (13) into Eq. (16), we obtain important expression for spectral distribution of the sub-holograms as follows:

$$
\begin{aligned}
\tilde{I}_{s}^{(j)}(\xi, y)= & \frac{2 \xi_{N}}{3} \sum_{n^{\prime}} \exp \left[-i 2 \pi n^{\prime} \frac{j}{3}\right]\left[B\left(\xi-n^{\prime} \frac{2 \xi_{N}}{3}, y\right)+C\left(\xi-n^{\prime} \frac{2 \xi_{N}}{3}-\xi_{0}, y\right)\right. \\
& \left.+C^{*}\left(\xi-n^{\prime} \frac{2 \xi_{N}}{3}+\xi_{0}, y\right)\right] \sum_{m} \delta(y-m d) \quad(i=0,1,2)
\end{aligned}
$$

This equation implies that spectral distribution of the hologram intensity is replicated at the spatial frequency of $n^{\prime} 2 \xi_{N} / 3$ and modulated in phase by $-\left(2 \pi n^{\prime} / 3\right) j$ depending on the replication order $n^{\prime}$. According to the interpolating procedure for missing data points in the method Toge et al. (2008), we simply observe spectra falling into the Nyquist base-band of the sub-sampling array, i.e., $|\xi|<\xi_{N} / 3$. Now we are a position to investigate the properties of the spectrum for various condition of the reference plane wave. 


\subsection{Considerations}

In the present subsection, we investigate effects of incident angle of the reference plane wave in the single-shot phase-shifting digital holography from spectral point of view based on Eq. (17).

First case is the case where Eq. (1) is satisfied. Corresponding spectral distribution is shown in Fig. 3(a). In this case, spectral components of the hologram falling into the Nyquist base-band, $|\xi| \leq \xi_{N} / 3$, are found to be $B$ of $n^{\prime}=0, C^{*}$ of $n^{\prime}=+1$, and $C$ of $n^{\prime}=-1$, and their locations are all at the origin of spectral coordinate. Therefore, the three sub hologram obtained in this case are give by

$$
\begin{array}{r}
\overline{I_{s}^{(0)}}(\xi, y)=\frac{2 \xi N}{3}\left[B(\xi, y)+C(\xi, y)+C^{*}(\xi, y)\right] \sum_{m} \delta(y-m d), \\
\overline{I_{s}^{(1)}}(\xi, y)=\frac{2 \xi_{N}}{3}\left[B(\xi, y)+C(\xi, y) \exp \left(i \frac{2 \pi}{3}\right)+C^{*}(\xi, y) \exp \left(-i \frac{2 \pi}{3}\right)\right] \sum_{m} \delta(y-m d), \\
\overline{I_{s}^{(2)}}(\xi, y)=\frac{2 \xi_{N}}{3}\left[B(\xi, y)+C(\xi, y) \exp \left(i \frac{4 \pi}{3}\right)+C^{*}(\xi, y) \exp \left(-i \frac{4 \pi}{3}\right)\right] \sum_{m} \delta(y-m d),
\end{array}
$$

where symbol $\cdots$ denotes filter out only low-frequency component less than $\xi=\xi_{N} / 3$. This is, in fact, performed by interpolating missing data due to sparse sampling in the space domain. As shown in Eqs.(18)-(20), important component $C$ in the each sub hologram has the relative phase difference of $2 \pi / 3$, which corresponds relative phase shift in the space domain representation of the sub holograms. Therefore we are able to simply apply the three-step algorithm in phase-shifting interferometry Schreiber et al. (2007) for calculating complex amplitude of the object wave at the image sensors, i.e., amplitude $a(x, y)$ and phase $\phi_{W}(x, y)$ (subscript ${ }_{W}$ denotes wrapped within $-\pi$ to $+\pi$ ),

$$
\begin{array}{r}
a(x, y)=\frac{1}{A}\left[\left\{\overline{I_{s}^{(1)}(x, y)}-\overline{I_{s}^{(2)}(x, y)}\right\}^{2}+\left\{\overline{2 I_{s}^{(0)}(x, y)}-\overline{I_{s}^{(1)}(x, y)}-\overline{I_{s}^{(2)}(x, y)}\right\}^{2}\right]^{1 / 2}, \\
\left.\phi_{W}(x, y)=\tan ^{-1}\left[\overline{\overline{2(0)}} \overline{\left.\overline{I_{S}^{(0)}(x, y)}-\overline{I_{s}^{(1)}(x, y)}-\overline{I_{s}^{(2)}(x, y)}\right\}}\right] . \overline{I_{s}^{(2)}(x, y)}\right]
\end{array}
$$

Next, we consider the case where Eq.(1) is not satisfied. Figure 3(b) shows the case where $\sin \theta>\lambda / 3 d$. Spectral components of the hologram falling into the Nyquist base-band, $|\xi| \leq$ $\xi_{N} / 3$, are the same as the previous case but their locations are different from the origin. Taking account this fact, the three sub holograms obtained in this case are given by

$$
\begin{array}{r}
\overline{I_{s}^{(0)}}(\xi, y)=\frac{2 \xi_{N}}{3}\left[B(\xi, y)+C\left\{\xi-\left(\xi_{0}-\frac{2 \xi_{N}}{3}\right), y\right\}+C^{*}\left\{\xi+\left(\xi_{0}-\frac{2 \xi_{N}}{3}\right), y\right\}\right] \\
\times \sum_{m} \delta(y-m d)
\end{array}
$$




$$
\begin{aligned}
\overline{I_{S}^{(1)}}(\xi, y) & =\frac{2 \xi_{N}}{3}\left[B(\xi, y)+C\left\{\xi-\left(\xi_{0}-\frac{2 \xi_{N}}{3}\right), y\right\} \exp \left(i \frac{2 \pi}{3}\right)\right. \\
+ & \left.C^{*}\left\{\xi+\left(\xi_{0}-\frac{2 \xi_{N}}{3}\right), y\right\} \exp \left(-i \frac{2 \pi}{3}\right)\right] \sum_{m} \delta(y-m d), \\
\overline{I_{S}^{(2)}}(\xi, y) & =\frac{2 \xi_{N}}{3}\left[B(\xi, y)+C\left\{\xi-\left(\xi_{0}-\frac{2 \xi_{N}}{3}\right), y\right\} \exp \left(i \frac{4 \pi}{3}\right)\right. \\
+ & \left.C^{*}\left\{\xi+\left(\xi_{0}-\frac{2 \xi_{N}}{3}\right), y\right\} \exp \left(-i \frac{4 \pi}{3}\right)\right] \sum_{m} \delta(y-m d) .
\end{aligned}
$$

As shown in Eqs. (23)-(25), phase-shift relationship between these three sub holograms still remains but the components $C(\xi, y)$ and $C^{*}(\xi, y)$ are modulated by the spatial linear carrier with spatial frequency of $\xi_{0}-2 \xi_{N} / 3$. It comes from the misalignment of the reference plane wave. Therefore, simple application of the three-step algorithm given by Eqs. (21) and (22) will lead the wrong result. For the case of the color digital holography based on the single-shot method proposed by Sato et al. Toge et al. (2008), the angle of the incident satisfying Eq. (1) is inevitably different with different color of the light. Therefore, in such case, one must align the optical components for each color of the reference waves independently. This suggests that the color digital holography by Sato et al. Toge et al. (2008) seems to be done with very careful and technical procedures. The simple way to obtain the correct complex amplitude of the object wave at the image sensor without any careful alignment is to compensate the phase due to linear carrier with the frequency of $\xi_{0}-2 \xi_{N} / 3$ after unwrapping the phase obtained by using Eq. (22). More concretely, correct phase distribution is obtained by subtracting the following phase:

$$
2 \pi\left[\xi_{0}-\frac{2 \xi_{N}}{3}\right] \text { nd } \quad(n=0,1,2, \cdots) .
$$

from the unwrapped phase at each pixel. For linear-phase compensation, we have to measure additional hologram without object and derive the frequency of the linear carrier before or after the measurement of object wave.

Another extreme case where Eq. (1) is not satisfied is shown in Fig. 3(c). In this case the incident angle, $\theta$, is too small for components Cs of the higher order of $n^{\prime}$ to fall into the Nyquist base-band, $|\xi| \leq \xi_{N} / 3$. Therefore, there is no phase-shifting relationship between three sub holograms obtained from single off-axis hologram, and therefore, reconstruction of object waves using Eqs. (21) and (22) will be in vain both amplitude and phase. One solution to derive the correct complex amplitude in the present case is to use of the Fourier transform technique Takeda et al. (1982).

\section{Verification by computer simulations}

In order to demonstrate the present theoretical analysis, computer simulations are performed for each case discussed in the previous section. The object wave at the image sensor considered here is given by

$$
U_{O}(x, y)=\exp \left[\mathrm{i} 2 \pi\left(x^{2}+y^{2}\right)^{2}\right] \quad(|x|,|y| \leq 1[\mathrm{~mm}]),
$$

i.e., the phase object is assumed. Total number of pixels of the image sensor in the horizontal and vertical directions is 256 . Therefore pixel size, $d$, is $7.8 \mu \mathrm{m}$. Figures 4 (a) and (b) show a 


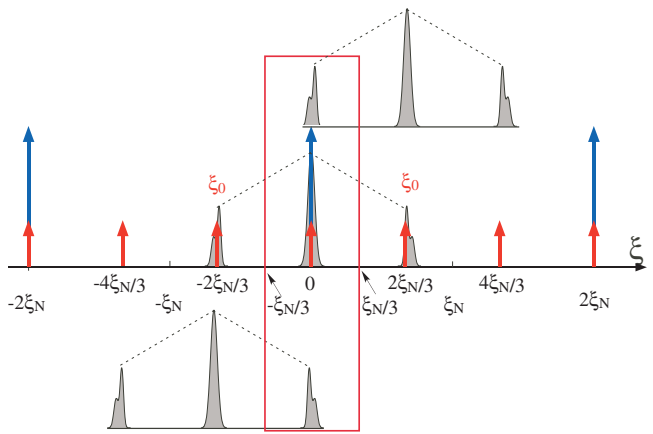

(a) $\sin \theta=\lambda / 3 d, \quad \xi_{0}=1 / 3 d=2 \xi_{N} / 3$

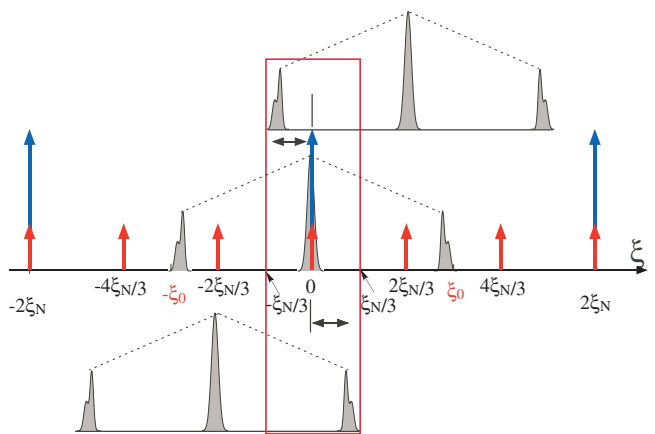

(b) $\lambda / 2 d>\sin \theta>\lambda / 3 d, 1 / 2 d=\xi_{N}>\xi_{0}>1 / 3 d=2 \xi_{N} / 3$

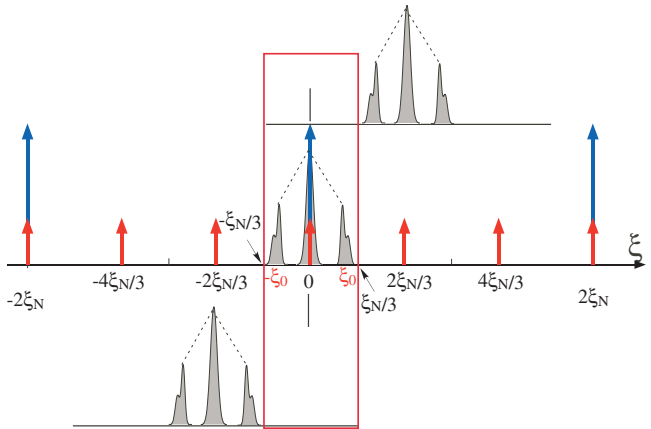

(c) $\sin \theta<\lambda / 6 d, \quad \xi_{0}<1 / 6 d=\xi_{N} / 3$

Fig. 3. Spectral distributions of resultant sub-holograms for various conditions of the incident angle of the reference plane wave. (a) $\sin \theta=\lambda / 3 d$ and $\xi_{0}=1 / 3 d=2 \xi_{N} / 3$, (b) $\sin \theta>\lambda / 3 d$ and $\xi_{0}>1 / 3 d=2 \xi_{N} / 3$, and (c) $\sin \theta<\lambda / 6 d$ and $\xi_{0}<1 / 6 d=\xi_{N} / 3$, respectively. 
phase distribution of the object wave under test in gray level and its intensity distribution of a hologram recorded by in-line geometry, respectively.

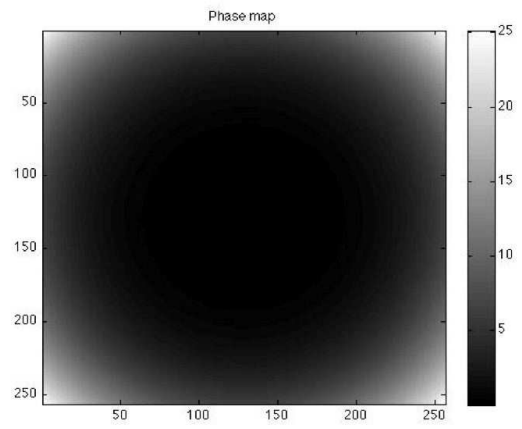

(a)

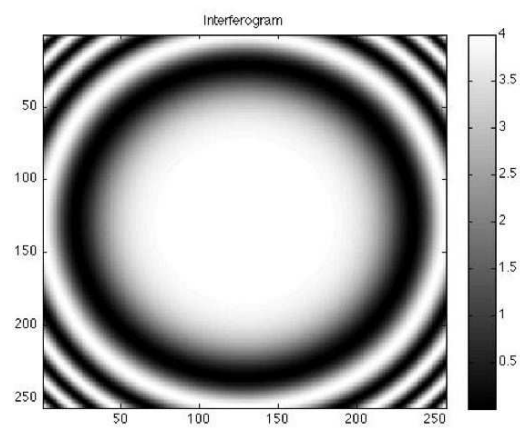

(b)

Fig. 4. Object wave at the image sensor investigated in computer simulation: (a) phase distribution in gray level and (b) intensity distribution of a hologram obtained by the in-line geometry.

Figures 5(a)-(c) show intensity distributions of holograms detected by the image sensor in the off-axis recording geometry shown in Fig. 1 (a) with an angle of (a) $\sin \theta=\lambda / 3 d$, (b) $\sin \theta=1.4 \lambda / 3 d>\lambda / 3 d$, and (c) $\sin \theta=0.8 \lambda / 6 d<\lambda / 6 d$, respectively. Corresponding spatial carrier frequency introduced by tilted reference plane waves are (a) $\xi_{0}=1 / 3 d=2 \xi_{N} / 3$, (b) $\xi_{0}=1.4 / 3 d=2.8 \xi_{N} / 3$, and (c) $\xi_{0}=0.8 / 6 d=0.8 \xi_{N} / 3$, respectively. Corresponding spectral relations are schematically given in Figs. 3(a), (b), and (c), respectively. In the case of the Figs.5 (a) and (b), where side-band spectra of the higher order $\left(n^{\prime}= \pm 1\right)$ falls in to the Nyquist base-band of $|\xi| \leq \xi_{N} / 3=1 / 6 d$ [see Figs.3(a) and (b)], intensity distributions of the detected holograms contain aliased or moiré fringes. In contrast to them, Fig. 5 (c) shows clear interference fringes with linear carrier because there are no higher order side-band spectra in the Nyquist base-band [see Fig. 3(c)], and therefore, no moiré fringes.

Figures 5(a1)-(a3) denote sub-holograms obtained from the Fig. 5(a) by sparsely sampling with the interval of $3 d$. Each of them shows similar coarse fringe pattern those obtained in the in-line geometry as shown in Fig. 4(b). Furthermore, their coarse fringes are shifted in their phase $2 \pi / 3$ each other. This fact confirms that phase distribution of the object wave at the image sensor can be evaluated directly by using Eq.(22) after interpolating process.

Figures 5(b1)-(b3) show sub-holograms obtained from the Fig. 5(b) by using the same manner. The coarse fringes in these figures are different shape from those of the in-line holograms shown in Figs. 5(a1)-(a3) since there are carrier fringes with a spatial frequency of $\xi_{0}^{\prime}=\mid \xi_{0}-$ $2 \xi_{N} / 3|=| 2.8 \xi_{N} / 3-2 \xi_{N} / 3 \mid=0.8 \xi_{N} / 3$ due to large amount of tilt in the reference plane wave. However, it must be noted that there is the phase-shift relation between these coarse fringes with the phase value of $2 \pi / 3$ each other. Therefore, after interpolating process we obtain three phase-shift fringes which are available to calculate a phase distribution where the phase of object wave and linear phase of $\xi_{0}^{\prime} n d=\left(0.6 \xi_{N} / 3\right) n d(n=1,2,3, \ldots)$ are added. Final phase distribution of the object wave at the image sensor can be obtained by subtracting the spatial linear phase $\xi_{0}^{\prime} n d=\left(0.6 \xi_{N} / 3\right) n d(n=1,2,3, \ldots)$. This result supports our derived 


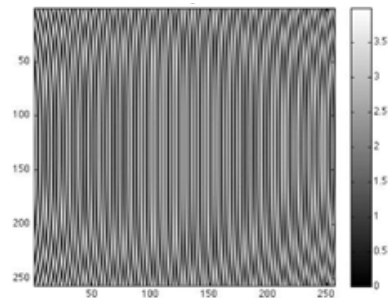

(a)

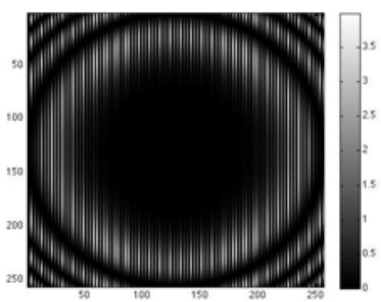

(a1)

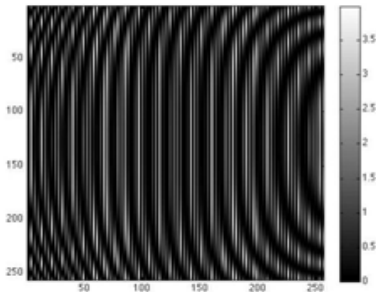

(b1)

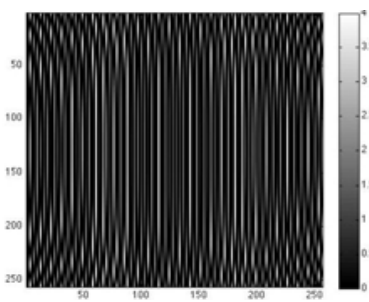

(c1)

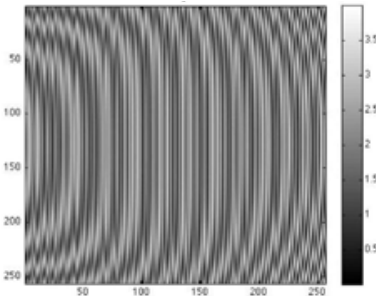

(b)

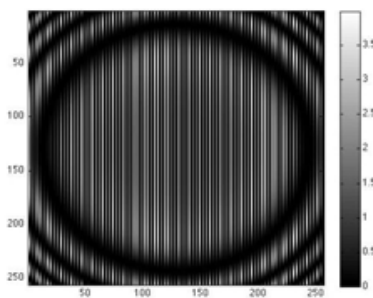

(a2)

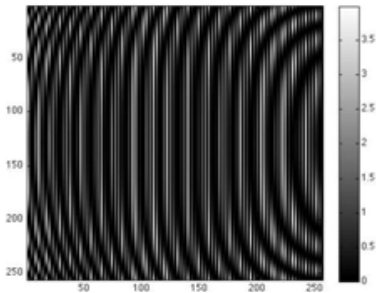

(b2)

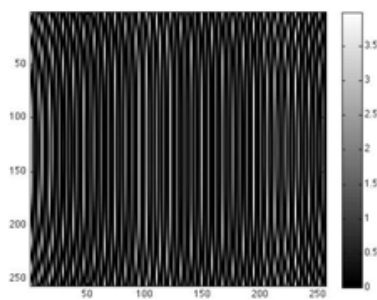

(c2)

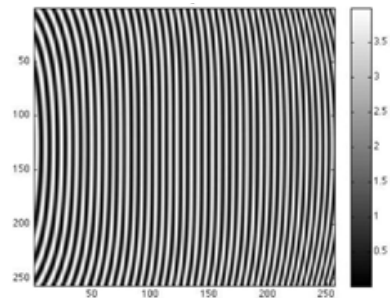

(c)

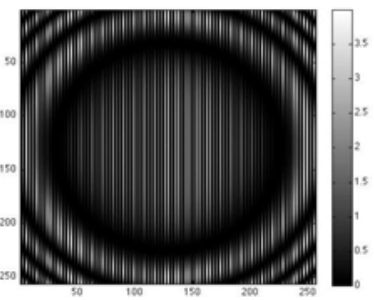

(a3)

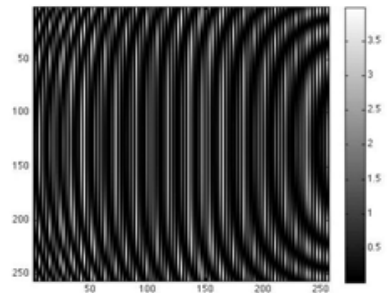

(b3)

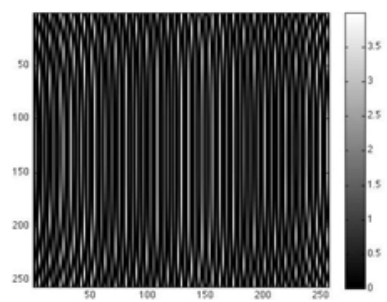

(c3)

Fig. 5. Typical results of the computer simulations: (a) interference fringes, $I_{S}(x, y)$, for $\sin \theta=\lambda / 3 d$ and $\xi_{0}=1 / 3 d=2 \xi_{N} / 3$, (b) interference fringe, $I_{S}(x, y)$, for $\sin \theta=1.4 \lambda / 3 d>\lambda / 3 d$ and $\xi_{0}=1.4 / 3 d=2.8 \xi_{N} / 3$, (c) interference fringe, $I_{S}(x, y)$, for $\sin \theta=0.8 \lambda / 6 d<\lambda / 6 d$ and $\xi_{0}=0.8 / 6 d=0.8 \xi_{N} / 3$, (a1)-(a3) corresponding sub-holograms $I_{s}^{(0)}, I_{s}^{(1)}, I_{s}^{(2)}$ for $\sin \theta=\lambda / 3 d$, (b1)-(b3) corresponding sub-holograms $I_{s}^{(0)}, I_{s}^{(1)}, I_{s}^{(2)}$ for $\sin \theta=1.4 \lambda / 3 d>\lambda / 3 d$, and (c1)-(c3) corresponding sub-holograms $I_{s}^{(0)}, I_{s}^{(1)}, I_{s}^{(2)}$ for $\sin \theta=0.8 \lambda / 6 d<\lambda / 6 d$. 
equations and procedures, i.e., Eqs.(23)-(25) and compensation of the linear phase given by Eq. (26), for the case where the condition Eq. (1) is not satisfied.

Figures 5(c1)-(c3) show sub-holograms from the Fig. 5(c). In contrast to previous two cases, there are no phase-shifting relation between each sub-hologram. This result comes from the fact that there are no aliasing, in other word, no moire fringe in the obtained hologram shown in Fig. 5(c). As predicted by our theoretical analysis we are not able not derived the phase map of the object wave at the image sensor by using the phase-shifting algorithm in this case. It will be done by using Fourier transform method Takeda et al. (1982) with the single-shot hologram of Fig. 5(c).

\section{Conclusions}

We have investigated theoretically tolerance of the incident angle of plane reference wave in space-division multiplexed single-shot phase-shifting digital holography presented by Sato et al Toge et al. (2008). It is found from our analysis that the rigorous alignment of the incident angle of the plane reference wave is very important requirement to reconstruct the correct complex amplitude of the object wave by direct application of the phase-shifting algorithm. It is also found that even if the incident angle of the plane reference wave departs from the condition given by Eq. (1), we have phase-shifted sub holograms from the single digital hologram by sparsely sampling. However, the direct application of the phase-shifting algorithm for these phase-shifted holograms results in wrong complex amplitude especially in phase distribution. We described the way to obtain correct phase distribution in this case from our theoretical analysis. That is to subtract the linear phase distribution introduced by misalignment of the reference plane wave. This solution was first derived and brought to light by our theoretical analysis within the authors knowledge, and widen field of the single-shot phase-shifting digital holography using off-axis plane wave illuminations.

\section{References}

Awatsuji, Y.; Sasada, M.; Kubota, T. (2004). Parallel quasi-phase-shifting digital holography, Applied Physics Letters, Vol.85, No.6, 1069-1071.

Gaskill, J. (1978). Linear Systems, Fourier Transforms, and Optics, John Wiley \& Sons, ISBN:0-471-29288-5, New York.

Harada, Y.; Goto, H.; Iwaki, T.; Wakaguri, S. (2008). Single-shot phase-shifting digital holography based on the spatial carrier interferometry, Extended Abstract on Optics E Photonics Japan 2008, Optical Society of Japan (Japan Society of Applied Physics), Tsukuba, Japan, pp.284-285. (in Japanese).

Harada, Y.; Goto, H.; Wan, A. (2009). Spatially-multiplexed single-shot phase-shifting digital holography based on the spatial carrier interferometry, Holographic Display Artists and Engineers Club (HODIC) Circular, Optical Society of Japan (Japan Society of Applied Physics), Vol.20, No. 29, 35-44. (in Japanese).

Kreis, T. (2005). Handbook of Holographic Interferometry, Wiley VCH, ISBN:3-527-40546-1, Weinheim.

Nomura, T.; Murata, S.; Nitanai, E; Numata, T. (2006). Phase-shifting digital holography with a phase difference between orthogonal polarization, Applied Optics, Vol.45, No. $4873-4877$.

Schnars, U. \& Jueptner, W. (1978). Digital Holography, Springer, ISBN:3-540-21934-x, Berlin. 
Schreiber, H.; Bruning, J.H.; Greivenkamp, J.E. (2007). Phase Shifting Interferometry, in Maralaca, D., ed., Optical Shop Testing, Third Edition, John Wiley \& Sons, ISBN:978-0-471-48404-2, Hoboken, pp.547-666.

Takeda, M.; Ina, H.; Kobayashi, S. (1982). Fourier-transform method of fringe-pattern analysis for computer-based topography and interferometry, Journal of Optical Society of America, Vol.72, No.1, 156-160.

Toge, H.; Fujiwara, H.; Sato, K. (2008). One-shot digital holography for recording color 3-d images, Proceedings of SPIE, Vol.69120, 69120U-1 - 69120U-7.

Wyant, J.C. (2003). Dynamic Interferometry, Optics \& Photonics News Vol.14, No.4, 36-41.

Yamaguchi, I. \& Zhang, T. (1997). Phase-shifting digital holography, Optics Letters, Vol.22, No.16, 1268-1270. 
ADVANCED HOLOGRAPHY METROLOGY AND IMAGING

Edace by trabele Napdanowa

\author{
Advanced Holography - Metrology and Imaging \\ Edited by Dr Izabela Naydenova
}

ISBN 978-953-307-729-1

Hard cover, 374 pages

Publisher InTech

Published online 09, November, 2011

Published in print edition November, 2011

Advanced Holography - Metrology and Imaging covers digital holographic microscopy and interferometry, including interferometry in the infra red. Other topics include synthetic imaging, the use of reflective spatial light modulators for writing dynamic holograms and image display using holographic screens. Holography is discussed as a vehicle for artistic expression and the use of software for the acquisition of skills in optics and holography is also presented. Each chapter provides a comprehensive introduction to a specific topic, with a survey of developments to date.

\section{How to reference}

In order to correctly reference this scholarly work, feel free to copy and paste the following:

Yasuhiro Harada, Aizuddin Wan and Hiroyasu Sone (2011). Single-Shot Phase-Shifting Digital Holography Based on the Spatial Carrier Interferometry and Its Tolerance Analysis, Advanced Holography - Metrology and Imaging, Dr Izabela Naydenova (Ed.), ISBN: 978-953-307-729-1, InTech, Available from:

http://www.intechopen.com/books/advanced-holography-metrology-and-imaging/single-shot-phase-shiftingdigital-holography-based-on-the-spatial-carrier-interferometry-and-its-to

\section{INTECH}

open science | open minds

\section{InTech Europe}

University Campus STeP Ri

Slavka Krautzeka 83/A

51000 Rijeka, Croatia

Phone: +385 (51) 770447

Fax: +385 (51) 686166

www.intechopen.com

\section{InTech China}

Unit 405, Office Block, Hotel Equatorial Shanghai

No.65, Yan An Road (West), Shanghai, 200040, China

中国上海市延安西路65号上海国际贵都大饭店办公楼 405 单元

Phone: +86-21-62489820

Fax: +86-21-62489821 
(C) 2011 The Author(s). Licensee IntechOpen. This is an open access article distributed under the terms of the Creative Commons Attribution 3.0 License, which permits unrestricted use, distribution, and reproduction in any medium, provided the original work is properly cited. 